\title{
Uncoupled Hartree-Fock Calculations of the Polarizability and Hyperpolarizabilities of Nitrophenols
}

\author{
R. BURSI,* M. LANKHORST, and D. FEIL \\ Chemical Physics Department, University of Twente, P.O. Box 217, 7500 AE Enschede, \\ The Netherlands
}

Received 26 April 1994; accepted 5 November 1994

\begin{abstract}
The polarizability and hyperpolarizabilities of nitrophenols as model compounds for studying nonlinear optics have been investigated at the Hartree-Fock level of approximation by means of the Dalgarno Uncoupled Hartree-Fock (DUHF) or Sum Over Orbitals (SOO) method. The additive character and the charge transfer effects in $\alpha, \beta$, and $\gamma$ have been analyzed in terms of the $\sigma$ and $\pi$ molecular orbital contributions, the contribution of the individual $\pi$ molecular orbitals, and the contribution of the highest occupied and the lowest unoccupied molecular orbitals. Within the SOO approach, the reliability of the Two-Level Model has been tested and the influence of the rotation of the nitro group and of the presence of the intramolecular hydrogen bonding in ortho-nitrophenol have been studied. The results show that the present method is a reliable and efficient tool for the prediction of trends in the molecular polarizability and

hyperpolarizabilities of large molecules. (c) 1995 by John Wiley \& Sons, Inc.
\end{abstract}

\section{Introduction}

$\mathbf{T}$ he nonlinear optical behavior of organic systems finds its origin in the response of the molecular electronic charge distributions to the external application of an intense electric field. In spite of the rapid development of highly advanced experimental techniques, the origin and the mechanisms by which a molecule responds to the external application of an electric field have as yet not been understood clearly. ${ }^{1,2}$ Theoretically, several well-established methods exist which enable the calculation of the static and dynamic linear and

*Author to whom all correspondence should be addressed. nonlinear properties of molecules. ${ }^{3-19}$ Each approach has been shown to have its own merits and defects because of the unavoidable compromise between, on the one side, the level of the theory employed and the consequent accuracy of the calculation and, on the other side, the size of the system to be investigated and the computational capabilities. Unfortunately, even when sophisticated $a b$ initio calculations ${ }^{15,16}$ are performed on molecular systems of the size of para-nitroaniline, the inclusion of electron correlation does not seem to ensure a priori a quantitative agreement with the experimental results, and factors such as the solvent effects and the vibrational contributions ${ }^{20}$ are often invoked to rationalize the discrepancy between theory and experiment. 
In view of the huge amount of computing resources necessary to handle large molecules at the sophisticated level, there seems to be a need for a method that invokes more approximations but that still is able to yield reliable hyperpolarizabilities values. In the present work, the linear polarizability $\alpha$ and the nonlinear polarizabilities $\beta$ and $\gamma$ have been calculated via expressions which have been derived for a single determinant wave function from the original equations of Orr and Ward, the Sum Over States (SOS) equations. ${ }^{21}$ These expressions can be regarded, within the Dalgarno uncoupling formalism scheme, as the Uncoupled Hartree-Fock equations. ${ }^{22}$ Since these equations involve sums over occupied and virtual molecular orbitals rather than sums over molecular states, we like to call them the Sum Over Orbitals (SOO) equations. The SOO method has been rarely used before for the calculation of the (hyper)polarizabilities, $1,7,23,24$ and an analysis of the advantages and disadvantages of this approach has not been performed as yet.

The aim of the present work is to show that the SOO method can indeed be considered as a valuable tool for the investigation of the linear and nonlinear optical properties of molecules. To accomplish this task, we have chosen to test the SOO method on the benzene, nitrobenzene, phenol, and the ortho-, meta-, and para-nitrophenol molecules since they are model molecules in the field of nonlinear optics (NLO).

In this article we will first test the performance of the method by a comparison of the calculated and experimental data. Subsequently, the (hyper)polarizabilities will be analyzed in terms of the total $\sigma$ and $\pi$ molecular orbital contributions, ${ }^{25,26}$ the individual $\pi$ orbital contributions, and the effect of the relative positions of the substituents. In the NLO literature, the empirical Two-Level Model ${ }^{27}$ (TLM) is often employed to predict the hyperpolarizabilities since it is a simple model and, therefore, easy to apply to large molecular systems. We will analyze the performance of this empirical model within the SOO formalism. Finally, since the most stable conformation of ortho-nitrophenol is intramolecularly hydrogen bonded, we will look at the effect on the (hyper)polarizabilities of such an intramolecular interaction.

\section{Computational Methods}

The SOO expressions for the static and dynamic linear and nonlinear polarizabilities have been derived from the original SOS equations for a single determinant wave function, which is an exact solution of the approximated Hartree-Fock equation, and by the application of Slater transition dipole moment matrix elements rules. ${ }^{28}$

The equations for these (hyper)polarizabilities are as follows:

$$
\begin{aligned}
\alpha_{i j}\left(-\omega_{\sigma} ; \omega_{1}\right)=2 I_{\left(-\omega_{\sigma} ; \omega_{1}\right)} \sum_{a r} \frac{\left\langle r_{i}\right\rangle_{a r}^{\sigma}\left\langle r_{j}\right\rangle_{r a}^{1}}{\left(\epsilon_{r}-\epsilon_{a}-\omega_{1}\right)} \\
\beta_{i j k}\left(-\omega_{\sigma} ; \omega_{1}, \omega_{2}\right) \\
=-2 K I_{\left(-\omega_{a} ; \omega_{1}, \omega_{2}\right)} \\
\quad \times\left\{\sum_{a r s} \frac{\left\langle r_{i}\right\rangle_{a r}^{\sigma}\left\langle r_{k}\right\rangle_{r s}^{2}\left\langle r_{j}\right\rangle_{s a}^{1}}{\left(\epsilon_{r}-\epsilon_{a}-\omega_{\sigma}\right)\left(\epsilon_{s}-\epsilon_{a}-\omega_{1}\right)}\right. \\
\left.-\sum_{a b r} \frac{\left\langle r_{i}\right\rangle_{a r}^{\sigma}\left\langle r_{k}\right\rangle_{b a}^{2}\left\langle r_{j}\right\rangle_{r b}^{1}}{\left(\epsilon_{r}-\epsilon_{a}-\omega_{\sigma}\right)\left(\epsilon_{r}-\epsilon_{b}-\omega_{1}\right)}\right\}
\end{aligned}
$$


The sums run over the occupied $a, b, c$ and the virtual $r, s, t$ molecular orbitals. The numerators are characterized by products of electric dipole transition moments between occupied and/or virtual molecular orbitals, while the denominators contain the differences between the molecular orbital energies and the energies of the photons of the perturbing radiation fields. The summations are premultiplied by two factors: the $I$ operator and the $K$ factor. ${ }^{21} I$ is a permutation operator which, in the case of $\gamma$, for instance, permutes in each sum the indexes $i, j, k$, and $l$. This will give rise to 2 !, 3 ! and 4 ! permutations (averaged by a factor of 1 !, 2! and 3!) in each sum which appears in the expressions of $\alpha, \beta$, and $\gamma$, respectively. The superscript on the right-hand side of each transition dipole moment indicates that in each permutation the perturbing frequencies $\omega_{\sigma}, \omega_{1}, \omega_{2}$, and $\omega_{3}\left(\omega_{\sigma}=\omega_{1}+\omega_{2}+\omega_{3}\right)$ must be permuted simultaneously with the corresponding $i, j, k$, and $l$ indexes. The $K$ factor has a specific value for each possible combination of perturbing $\omega^{\prime}$ s and it ensures that in each specific case only unique permutations are calculated. As mentioned before, the electric dipole moment matrix elements have been obtained by applying Slater rules. Because of the one-electron nature of the perturbation, only transition moments between ground and singly excited or between singly and other singly excited configurations do not vanish in the expressions of $\alpha$ and $\beta$. In the expression of $\gamma$, doubly excited configurations mix because their matrix elements with the singly excited configurations do not vanish. Equations (1) through (3) have been implemented in a home-built program HYPERPOL.

The SOO method is an approximate method. As shown by Langhoff et al., ${ }^{22}$ SOO expressions are approximations of the coupled Hartree-Fock (CPHF) equations and of their uncoupled form. The approximation lies in the use of molecular orbital energies in the denominator, where the Coulomb and exchange two-electron integrals representing the self-consistent field (SCF) correction to the orbital energies and the additional energy corrections due to the perturbation are completely neglected. When only orbital energies are employed, as in the present case, the crudest approximation is applied and these expressions are referred to as the Dalgarno Uncoupled Hartree-Fock equations. It has been observed ${ }^{29}$ that the use of only the orbital energies, instead of the SCF energies in the denominators of eqs. (1), (2), and (3), introduces a systematic error in the calculation: Orbital energy differences are generally 10 to $20 \%$ bigger than the corresponding SCF ones, leading to smaller values for the (hyper)polarizabilities.

We have attempted to estimate the error by which the SOO formalism is affected with respect to the CPHF formalism, by comparing existing CPHF (hyper)polarizabilities calculations with our SOO values, obtained for the same small molecules, at the same geometries and basis sets levels. ${ }^{30}$ The results of such an analysis show that for these molecules the SOO method is quantitatively less accurate than the CPHF method by, on the average, $12 \%, 25 \%$, and $80 \%$ in the calculations of $\alpha$, $\beta$, and $\gamma$, respectively. It would seem, therefore, that these large deviations imply that considerable caution is required in the application of the SOO method. However, it should be remembered that even the CPHF method does not yield results which are quantitatively in agreement with the experimental data. Our results (vide infra) show, certainly qualitatively and in some cases even quantitatively, a correct prediction of the nonlinear optical behavior. More important, the present method allows for calculations on medium- to large-sized molecules, which are very hard to handle with CPHF techniques.

The SOO expressions involve sums over an infinite number of molecular orbitals. It is expected, therefore, that very large basis sets must be employed to obtain the most accurate $\mathrm{SOO}$ values. It has been shown that in the calculation of the nonlinear optical properties, the use of diffuse basis functions improves significantly the agreement between theory and experiment. ${ }^{31-33}$ In the present work we have performed a large number of calculations starting from the crudest STO-3G basis set, up to the double-zeta $(\mathrm{DZ})^{34}$ and triplezeta valence $(\mathrm{TZV})^{35,36}$ plus polarization functions basis sets in order to verify the effect of the choice of the basis set on the SOO hyperpolarizabilities calculations.

The molecular geometries, for which the (hyper)polarizabilities have been calculated, are the results of geometry optimizations performed at the 3-21G basis set level. All molecules are planar and are characterized by at least a mirror plane, which contains the molecular skeletons. These geometries are shown in Figure 1. The calculation of the (hyper)polarizabilities requires accurate geometries, because particularly the hyperpolarizabilities are sensitive to the geometrical parameters. The $3-21 \mathrm{G}$ basis set has been proven to be sufficiently accurate in the prediction of the equilibrium geometries of molecules containing firstrow atoms ${ }^{37,38}$ and, in particular, in the prediction 
(a)

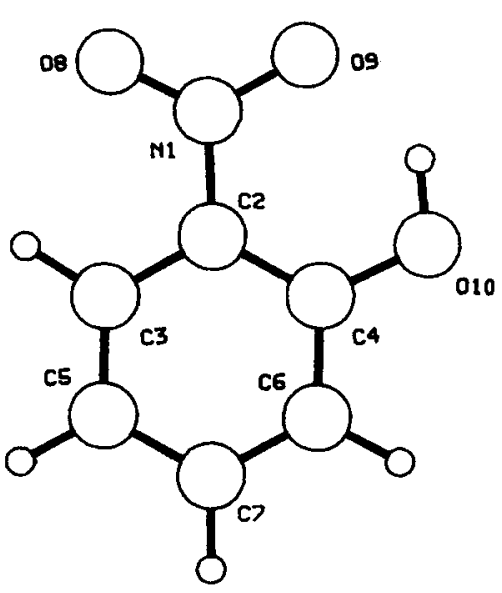

(c)

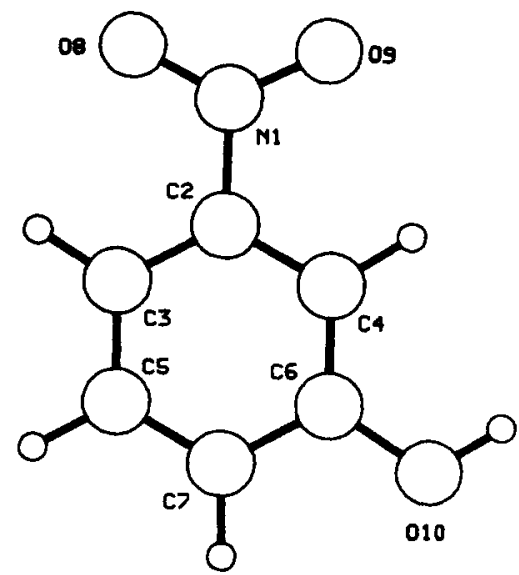

(b)

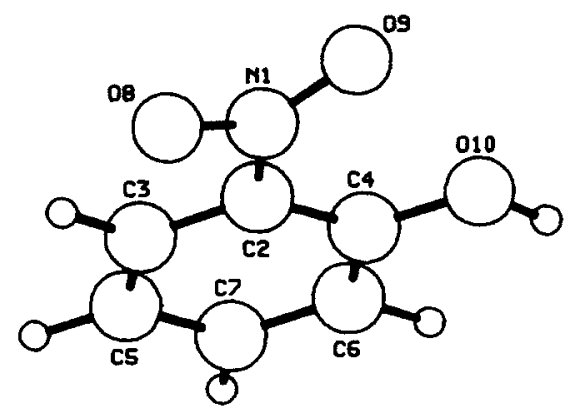

(d)

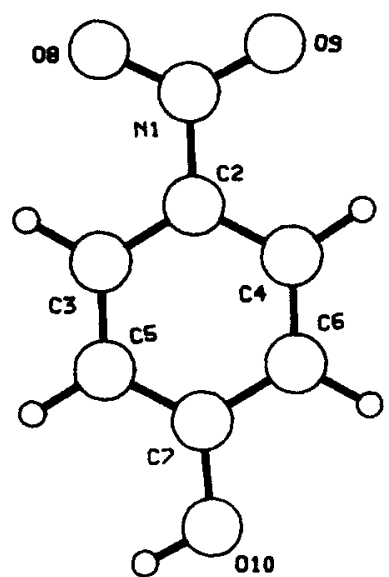

FIGURE 1. 3-21G optimized geometries of (a) ortho-nitrophenol (o-nph-a), $C_{s}$ symmetry, $E_{3-21 G}=-506.163$ au; (b) ortho-nitrophenol (o-nph-b), $C_{1}$ symmetry, $E_{3-21 G}=-506.140 \mathrm{au}$; (c) meta-nitrophenol, $C_{s}$ symmetry, $E_{3-21 G}=$ $-506.152 \mathrm{au}$; (d) para-nitrophenol, $C_{s}$ symmetry, $E_{3-21 G}=-506.155$. All molecules lie in the $x y$-plane.

of the conformation of the flexible orthonitrophenol, whose structure has been extensively studied, both experimentally and theoretically..$^{39,40}$ We have tested the quality of the 3-21G optimized geometry in the prediction of the (hyper)polarizabilities of these molecules by repeating the calculations of $\alpha, \beta$, and $\gamma$ of ortho-nitrophenol at a geometry optimized at the MP2 ${ }^{41}$ level of theory with the $6-31 \mathrm{G}^{*}$ basis set. The changes caused by the MP2/6-31G* geometry were found to be smaller than $2 \%$ in $\bar{\alpha}, \beta_{y}$, and $\bar{\gamma}$ and smaller than $3 \%$ in $\beta_{y}$. Because the major emphasis of the present work is on the relative comparison of the hyperpolarizabilities predicted for the different molecules and the minor emphasis is on the comparison between the theoretical and experimental values, the choice of the $3-21 \mathrm{G}$ basis set for the equilibrium geometries can be considered satisfactory. Major geometrical factors, like the rotation of the nitro group and the presence of the intramolecular hydrogen bond, will be analyzed in detail in the last part of this article.

The optimized equilibrium geometries have been checked by a force field calculation. We have performed the geometry optimizations, the calculations of the force fields, and the SCF calculations with the GAMESS (Daresbury) ${ }^{42}$ program. The Finite Field ${ }^{45}$ calculations have been performed with the GAMESS (Dakota) ${ }^{43}$ program. The GAMESS and the HYPERPOL calculations have been performed on a SG CHALLENGE L 2/128 and a CRAY Y-MP 4/464-supercomputer.

\section{Results}

We have calculated with the SOO method the values for the linear polarizability $\alpha$ and the non- 
linear hyperpolarizabilities $\beta$ and $\gamma$ of benzene, nitrobenzene, phenol, and the ortho-, meta-, and para-nitrophenols. The results are shown in Tables I through III. To investigate the basis set dependence of the method employed and the performance of each individual basis set within this formalism with respect to the experimental values, we have employed different basis sets: the crude Slater-type orbital basis set, the split valence and split valence plus polarization functions basis sets, the $\mathrm{DZ}$ and $\mathrm{TZV}$, and the $\mathrm{DZ}$ and $\mathrm{TZV}$ plus polarization functions basis sets DZP and TZVP, respectively.

The calculated static and dynamic values of the linear polarizability $\alpha$ are given in Table I. These results show a strong basis set dependence in two aspects: the dependence on the size of the basis set and the influence of the inclusion of polarization functions. Starting with the STO-3G results, it is observed that the values obtained with this basis set are generally more than $50 \%$ off from the experimental values. As expected, the split valence 3-21G and 6-31G basis sets result in more accurate values, though a comparison between these two basis sets shows that the larger number of primitives employed in the 6-31G calculation does not significantly affect the values for $\alpha$. Finally, Table I shows that the larger valence basis sets $\mathrm{DZ}$ and $\mathrm{TZV}$ produce quantitatively more accurate results.

The inclusion of polarization functions in either the split valence or in the DZ and TZV basis sets is observed to result in similar improvements: For all basis sets the calculated values for $\alpha$ become significantly closer to the experimental ones. On the other hand, the comparison between the $6-31 G^{*}$ and $6-31 G^{* *}$ results demonstrates that the use of polarization functions on the hydrogen atoms has only a very small effect. Though slightly smaller in size than the $6-31 \mathrm{G}^{*}$ basis set, it is noteworthy to observe that the TZV basis set produces results which are closer to experiment. We conclude that within the SOO formalism, $\alpha$, as a function of the number of basis functions, slowly converges to a limiting value.

Table I shows that the best results are obtained with the TZVP basis set. These results deviate from the experimental data on the average by about $20 \%$, and the $\bar{\alpha}(\omega)$ value of meta-nitrophenol is remarkably within $10 \%$ of the experimental value. Although the other basis sets result in larger deviations, it is gratifying to notice that all basis sets reproduce the experimental trends accurately.

TABLE I.

Theoretical and Experimental Linear Polarizabilities.

\begin{tabular}{|c|c|c|c|c|c|c|c|c|c|c|}
\hline & \multicolumn{9}{|c|}{ Basis sets } & \multirow[t]{2}{*}{ Exp. $^{a}$} \\
\hline & STO-3G & $3-21 G$ & $6-31 G$ & $6-31 G^{\star}$ & $6-31 \mathrm{G} * \star$ & $\mathrm{DZ}$ & TZV & $\mathrm{DZP}$ & TZVP & \\
\hline \multicolumn{11}{|l|}{ ben } \\
\hline $\bar{\alpha}^{\mathrm{b}}(0)$ & 31.60 & 47.29 & 49.36 & 51.93 & 52.59 & 50.83 & 56.00 & 55.41 & 59.54 & \\
\hline $\begin{array}{l}\bar{\alpha}(\omega) \\
\text { ph }\end{array}$ & 31.73 & 47.51 & 49.56 & 52.13 & 52.82 & 51.08 & 56.27 & 55.68 & 59.83 & 74.2 \\
\hline $\bar{\alpha}(0)$ & 34.33 & 49.68 & 51.89 & 55.06 & 55.72 & 53.60 & 58.78 & 58.74 & 62.57 & \\
\hline $\begin{array}{l}\bar{\alpha}(\omega) \\
\text { nben }\end{array}$ & 34.46 & 49.91 & 52.13 & 55.33 & 55.97 & 53.86 & 59.07 & 59.02 & 62.87 & 81.0 \\
\hline $\bar{\alpha}(0)$ & 44.89 & 61.79 & 64.76 & 67.95 & 68.46 & 66.51 & 71.85 & 71.71 & 75.31 & \\
\hline $\bar{\alpha}(\omega)$ & 45.11 & 62.11 & 65.09 & 68.27 & 68.79 & 66.87 & 72.23 & 72.07 & 75.69 & 94.5 \\
\hline \multicolumn{11}{|l|}{ o-nph } \\
\hline $\bar{\alpha}(0)$ & 48.82 & 65.44 & 68.62 & 72.18 & 72.68 & 70.44 & 75.92 & 75.99 & 79.41 & \\
\hline \multicolumn{11}{|l|}{ m-nph } \\
\hline $\bar{\alpha}(0)$ & 47.79 & 64.60 & 67.75 & 71.52 & 72.02 & 69.72 & 75.13 & 75.48 & 78.81 & \\
\hline $\bar{\alpha}(\omega)$ & 48.04 & 64.93 & 68.10 & 71.86 & 72.36 & 70.09 & 75.53 & 75.85 & 79.21 & 87.7 \\
\hline \multicolumn{11}{|l|}{ p-nph } \\
\hline $\bar{\alpha}(0)$ & 48.27 & 64.83 & 68.00 & 71.69 & 72.19 & 69.66 & 75.22 & 75.34 & 78.78 & \\
\hline $\bar{\alpha}(\omega)$ & 48.51 & 65.16 & 68.35 & 72.03 & 72.53 & 70.04 & 75.62 & 75.71 & 79.17 & 101.2 \\
\hline
\end{tabular}


BURSI, LANKHORST, AND FEIL

TABLE II.

Theoretical and Experimental First Hyperpolarizabilities.

\begin{tabular}{|c|c|c|c|c|c|c|c|c|c|c|}
\hline & STO-3G & $3-21 G$ & $6-31 G$ & $6-31 G^{*}$ & $\begin{array}{c}\text { Basis sets } \\
6-31 G^{\star \star}\end{array}$ & $D Z$ & TZV & DZP & TZVP & Exp. $^{a}$ \\
\hline \multicolumn{11}{|l|}{ ben } \\
\hline$\beta_{y}(0)^{b}$ & 0.00 & 0.00 & 0.00 & 0.00 & 0.00 & 0.00 & 0.00 & 0.00 & 0.00 & \\
\hline $\begin{array}{l}\beta_{y}(\omega) \\
\mathrm{ph}\end{array}$ & 0.00 & 0.00 & 0.00 & 0.00 & 0.00 & 0.00 & 0.00 & 0.00 & 0.00 & 0.00 \\
\hline$\beta_{y}(0)$ & -27.10 & -37.15 & -36.37 & -33.55 & -34.10 & -41.66 & -41.65 & -39.01 & -40.05 & \\
\hline $\begin{array}{l}\beta_{y}(\omega) \\
\text { nben }\end{array}$ & -28.24 & -39.35 & -38.39 & -34.66 & -35.24 & -42.28 & -43.17 & -39.55 & -41.58 & $<-23.0$ \\
\hline$\beta_{y}(0)$ & -46.03 & -76.85 & -93.17 & -77.58 & -77.41 & -92.36 & -87.24 & -78.11 & -75.65 & \\
\hline $\begin{array}{l}\beta_{y}(\omega) \\
0-n p h\end{array}$ & -48.08 & -81.08 & -94.48 & -81.92 & -82.41 & -98.50 & -100.60 & -83.26 & -88.39 & -219.9 \\
\hline$\beta_{y}(0)$ & -62.92 & -91.27 & -94.13 & -81.34 & -81.54 & -93.97 & -93.88 & -83.16 & -85.42 & \\
\hline $\begin{array}{l}\beta_{y}(\omega) \\
\text { m-nph }\end{array}$ & -64.64 & -93.80 & -97.01 & -83.91 & -83.67 & -96.98 & -96.86 & -85.94 & -88.30 & -138.9 \\
\hline$\beta_{y}(0)$ & -36.70 & -63.77 & -82.71 & -55.74 & -55.68 & -65.41 & -62.75 & -54.21 & -53.93 & \\
\hline $\begin{array}{l}\beta_{y}(\omega) \\
\text { p-nph }\end{array}$ & -38.84 & -68.91 & -89.07 & -59.84 & -59.79 & -70.80 & -68.91 & -58.47 & -58.33 & -92.6 \\
\hline$\beta_{y}(0)$ & -106.86 & -160.28 & -177.27 & -150.63 & -151.04 & -177.63 & -179.72 & -153.99 & -158.85 & \\
\hline$\beta_{y}(\omega)$ & -112.39 & -170.67 & -188.73 & -160.07 & -160.52 & -189.26 & -191.62 & -163.88 & -169.47 & -347.3 \\
\hline
\end{tabular}

$\beta_{y}$ is given in au. The $\beta_{y}$ values have been calculated with respect to the direction of the molecular electric dipole moments. ${ }^{a}$ See ref. 46 .

${ }^{\mathrm{b}} \beta_{i}=\frac{1}{3}\left(\beta_{i j i}+2 \beta_{i j}\right) ; \beta_{i}(2 \omega ; \omega, \omega), \omega=0.04282 \mathrm{au}=1.064 \mu \mathrm{m} . K(-2 \omega ; \omega, \omega)=\frac{1}{2}$.

TABLE III.

Theoretical and Experimental Second Hyperpolarizabilities.

\begin{tabular}{|c|c|c|c|c|c|c|c|c|c|c|}
\hline & STO-3G & $3-21 G$ & $6-31 G$ & $6-31 G^{\star}$ & $\begin{array}{c}\text { Basis sets } \\
6-31 G^{\star *}\end{array}$ & $\mathrm{DZ}$ & TZV & DZP & TZVP & Exp. $^{a}$ \\
\hline \multicolumn{11}{|l|}{ ben } \\
\hline $\bar{\gamma}^{b}(0)$ & 163.10 & 352.86 & 423.98 & 401.60 & 409.65 & 568.38 & 901.29 & 549.34 & 933.06 & \\
\hline $\begin{array}{l}\bar{\gamma}(\omega) \\
\text { ph }\end{array}$ & 169.35 & 367.87 & 441.57 & 418.95 & 427.47 & 593.92 & 942.86 & 575.18 & 977.81 & 6155.1 \\
\hline $\bar{\gamma}(0)$ & 277.00 & 481.79 & 548.02 & 511.70 & 519.87 & 709.82 & 1036.40 & 674.35 & 1054.35 & \\
\hline $\begin{array}{l}\bar{\gamma}(\omega) \\
\text { nben }\end{array}$ & 288.77 & 503.12 & 571.76 & 534.20 & 542.84 & 742.54 & 1084.85 & 706.52 & 1105.19 & 7942.1 \\
\hline $\bar{\gamma}(0)$ & 446.69 & 988.10 & 1169.03 & 1059.41 & 1067.34 & 1345.40 & 1660.98 & 1256.11 & 1632.68 & \\
\hline $\bar{\gamma}(\omega)$ & 475.43 & 1049.42 & 1262.36 & 1138.72 & 1143.23 & 1443.37 & 1774.71 & 1344.01 & 1740.92 & 81009.0 \\
\hline \multicolumn{11}{|l|}{ o-nph } \\
\hline $\bar{\gamma}(0)$ & 716.39 & 1280.12 & 1453.94 & 1289.33 & 1297.91 & 1635.81 & 1930.37 & 1486.02 & 1846.43 & \\
\hline$\dot{\gamma}(\omega)$ & 766.84 & 1377.31 & 1569.86 & 1385.01 & 1394.29 & 1763.15 & 2072.51 & 1595.35 & 1976.22 & 5956.5 \\
\hline \multicolumn{11}{|l|}{ m-nph } \\
\hline $\bar{\gamma}(0)$ & 585.75 & 1180.97 & 1372.73 & 1225.20 & 1233.89 & 1569.11 & 1878.92 & 1439.58 & 1817.99 & \\
\hline $\bar{\gamma}(\omega)$ & 624.70 & 1264.05 & 1474.38 & 1316.83 & 1326.18 & 1688.90 & 2014.51 & 1544.92 & 1944.42 & 11913.1 \\
\hline \multicolumn{11}{|l|}{ p-nph } \\
\hline $\bar{\gamma}(0)$ & 893.24 & 1589.87 & 1803.29 & 1567.89 & 1576.85 & 1985.99 & 2292.57 & 1771.85 & 2185.65 & \\
\hline $\bar{\gamma}(\omega)$ & 957.61 & 1721.37 & 1959.19 & 1694.17 & 1703.85 & 2152.44 & 2475.62 & 1912.10 & 2309.21 & 15884.1 \\
\hline
\end{tabular}

$\bar{\gamma}$ is given in au.

See ref. 46 .

${ }^{\mathrm{b}} \bar{\gamma}=\frac{1}{15}\left(2 \gamma_{i j i j}+\gamma_{i j i i}\right) ; \bar{\gamma}(2 \omega ; \omega, \omega, 0), \omega=0.04282 \mathrm{au}=1.064 \mu \mathrm{m} . K(-2 \omega ; \omega, \omega, 0)=\frac{3}{2}$. 
The static and dynamic polarizabilities values, calculated in conditions far from resonance, do not differ significantly within each basis set, and the difference between the two values is only slightly sensitive to the employed basis set.

In Table II, the calculated and experimental values of the first hyperpolarizability $\beta_{y}$ are shown. Because benzene is a centrosymmetric molecule, each component of the $\beta$ tensor identically vanishes. A first analysis of the calculated $\beta_{y}$ values and their comparisons to the corresponding experimental values reveals the different character of the first hyperpolarizability $\beta$ with respect to the linear polarizability $\alpha$ and shows that it is more difficult to obtain a quantitative estimate of $\beta$. It is observed that the calculation of $\beta$ is very sensitive to the basis set employed and that it does not seem to converge to a limiting value as a function of the number of basis functions. Such a behavior is also observed in CPHF calculations of $\beta^{44}$

The values calculated with the STO-3G basis set indicate that this basis set is unsuitable for a calculation of $\beta$. Unexpectedly, there do not seem to be large differences between the split valence $6-31 \mathrm{G}$ and the DZ and TZV basis sets results. In contrast to what has been found for $\alpha$, the different number of primitives between the 3-21G and $6-31 \mathrm{G}$ basis sets does affect the calculation of $\beta$. The addition of polarization functions to either the 6-31G basis or the DZ or TZV does not increase the $\beta$ values but diminishes them.

Quantitatively, the best $\beta$ values have been obtained with the DZ and TZV basis sets. If we compare these values to the corresponding experimental values, the discrepancy between theory and experiment is, on the average, $40 \%$, with the exception of phenol, for which the predicted $\beta$ values generally overestimate the experimental data. The $6-31 \mathrm{G} \beta$ values are also close to the experimental results, but the relative magnitude of the experimental ortho, meta, and para values is not reproduced by the 6-31G calculations with the same accuracy as by the DZ or TZV calculations.

Qualitatively, the experiment yields a negligible value of $\beta$ for phenol and a very large value of $\beta$ for nitrobenzene. In the disubstituted compounds, the para position is the most favorable to the charge transfer effects, which are of main importance for $\beta .^{27}$ The ortho position of the substituents is favorable, but not as much as the para isomer and, finally, the meta position is the least favorable. Focusing on the TZV results, the experimental trend is reproduced by the calculations, with nitrobenzene being the only exception. Phenol has the smallest $\beta$ value, while paranitrophenol has the largest one, but for nitrobenzene the calculations underestimate $\beta$. Finally, the dispersion contribution to the $\beta$ values is, on the average, reproduced systematically by the calculation through all basis sets for each molecule and for a given basis set the difference between static and dynamic values increases in going from phenol to para-nitrophenol.

In Table III, the values calculated for the second hyperpolarizability $\gamma$ are given. The STO-3G values are extremely poor with respect to the larger basis sets and experimental values, while the split valence basis sets provide better results. As was found for $\beta$, in the calculation of $\gamma$ the number of primitives makes a considerable difference between the 3-21G and the 6-31G calculated $\gamma$ values, the latter being larger than the former ones. The addition of polarization functions on the heavy atoms decreases the 6-31G $\gamma$ values, as was observed in the calculation of $\beta$, while the polarization functions on the hydrogen atoms increase the 6-31G* values, similarly to the calculations of $\alpha$. The values obtained with DZ and TZV basis sets are the largest ones and in best agreement with the experimental values. The TZV and the $6-31 \mathrm{G}^{*}$ basis sets are characterized by almost the same number of basis functions, but the TZV basis produces significantly better results. The addition of polarization functions on the DZ and TZV basis sets generally decreases the magnitude of the $\gamma$ values.

The best agreement between theory and experiment is obtained by the TZV basis set, which, on the average, produces $\gamma$ values which are $80 \%$ off from the experimental values. While the TZV $\gamma$ value of nitrobenzene is largely underestimated, the TZV value of the ortho isomer is reproduced within a $65 \%$ error from the experiment. The experimental trend in the present case is closely reproduced by the calculation, with the only exception being the relative magnitude of the ortho and meta isomers. The observation that the experimental trend is well reproduced is in agreement with the conclusions of a study on the second hyperpolarizability of mono- and di-substituted benzenes by the SOO method by Tomonari et al. ${ }^{23}$ These results show that the SOO method can become useful in a preliminary qualitative investigation of the second hyperpolarizabilities of mediumto large-sized molecules.

In a first attempt to provide a partial conclusion from the analysis of the results shown in Tables I through III, it is observed that the SOO method 
has produced for this set of molecules $\alpha, \beta$, and $\gamma$ values within $20 \%, 40 \%$, and $80 \%$ of the corresponding experimental values. It is noteworthy that both $\beta$ and $\gamma$ theoretical values of nitrobenzene have shown the largest discrepancy with the experimental values while the best agreement between theoretical and experimental $\beta$ and $\gamma$ values has been obtained for the ortho and meta isomers, respectively. The $\mathrm{SOO}$ results on nitrobenzene are not surprising, however, because even the more accurate CPHF $\beta$ and $\gamma$ values of nitrobenzene differ from the experimental results by more than $50 \%$ and $80 \%$, respectively. ${ }^{44}$ Part of these discrepancies may well be due to solvent effects.

Finally, to provide a more detailed analysis of the performance on these molecules of the SOO method with respect to the CPHF method, we have calculated the linear polarizability $\alpha$ and the nonlinear hyperpolarizability $\beta$ of nitrobenzene, phenol, and the ortho-, meta-, and paranitrophenols via the finite field (FF) method, ${ }^{45}$ which is equivalent to the CPHF method at the Hartree-Fock level of theory. The linear and nonlinear polarizabilities $\alpha$ and $\beta$ have been calculated at the TZVP and DZ basis set levels, respectively, since with these basis sets the best SOO $\alpha$ and $\beta$ values have been obtained. As is shown in Table IV, the $\alpha_{y y}(S O O)$ components are smaller than the $\alpha_{y y}(F F)$ components by about $10 \%$, and this result is consistent with the results which have been obtained in our previous comparison of the SOO and the CPHF methods. Unfortunately, on the average the $\beta_{y \psi y}(S O O)$ components are smaller than the $\beta_{y y y}(F F)$ components by about $60 \%$, which seems to indicate that in mono- and di-substituted benzenes the discrepancy between the FF and the SOO methods becomes larger. It is noteworthy, however, to observe that the FF calculations at the DZ basis set level fail to reproduce an accurate estimate of the $\beta_{y y y}$ component of nitrobenzene and that they reproduce for the remaining molecules $\beta_{y y y}$ components which are large with respect to the experimental values of $\beta_{y}$.

The FF method is a method which is superior to the SOO method because it is self-consistent with respect to the perturbed orbitals. The FF method, however, is limited to static fields and thus cannot yield dispersion. Neither do analytical methods exist to find the mechanisms which drive the nonlinear response. These limitations do not affect the less accurate SOO method, which, in a straightforward way, allows the calculation of the frequencydependent hyperpolarizabilities and the interpretation of the hyperpolarizabilities values in terms of each individual molecular orbital contribution.

\section{Discussion}

The SOO expressions of the polarizability $\alpha$ and of the hyperpolarizabilities $\beta$ and $\gamma$ involve sums over occupied and virtual orbitals [see eqs. (1) through (3)]. Consequently, it is always possible to analyze at the molecular orbital level these molecular properties. In this section we will be concerned with investigating the polarizability $\alpha$ and the hyperpolarizabilities $\beta$ and $\gamma$ in terms of $\sigma$ and $\pi$ molecular orbital contributions $s^{25,26}$ and individual $\pi$ molecular orbital contributions. Since within the SOO approach the TZV basis set has produced the closest values to the experimental $\beta$ and $\gamma$ values, we will perform such a discussion on the TZV results. Subsequently, we will analyze the results in terms of the Two-Level Model (TLM) and in terms of the highest occupied and the lowest unoccupied molecular orbital, HOMO and LUMO, respectively. Finally, the effect of the intramolecular hydrogen bond on the

TABLE IV.

Comparison between the Sum Over Orbitals (Static Case)

and the Finite Field $\left(E_{y}=0.2 \mathrm{~V} / \AA=3.88937 \times 10^{-3}\right.$ au) Methods.

\begin{tabular}{lrrrrr}
\hline & nben & \multicolumn{1}{c}{ ph } & o-nph & m-nph & p-nph \\
\hline$\alpha_{y y}(S O O)^{\mathrm{a}}$ & 87.19 & 74.90 & 95.66 & 92.83 & 95.51 \\
$\alpha_{y y}(F F)$ & 98.54 & 80.35 & 100.52 & 101.95 & 111.81 \\
$\beta_{y y y}(S O O)^{\mathrm{b}}$ & -60.04 & -33.22 & -91.77 & -63.84 & -131.98 \\
$\beta_{y y y}(F F)$ & -172.31 & -73.86 & -257.26 & -185.22 & -400.36 \\
\hline
\end{tabular}

${ }^{a}$ TZVP basis set.

${ }^{\mathrm{b}} \mathrm{DZ}$ basis set. 
(hyper)polarizabilities of orthonitrophenol will be investigated.

Benzene and nitrobenzene possess $D_{6 h}$ and $C_{20}$ symmetry, respectively, while phenol and the ortho-, meta-, and para-nitrophenols are characterized by $C_{s}$ symmetry. Due to the symmetry, the separation into $\sigma$ and $\pi$ contributions is exact for all in-plane diagonal and off-diagonal components, while it is a good approximation for all off-plane diagonal and off-diagonal components of the tensors, since the transition moments between $\sigma\left(\sigma^{*}\right)$ and $\pi\left(\pi^{*}\right)$ orbitals are very small in comparison with the transition moments between $\sigma(\pi)$ and $\sigma^{*}\left(\pi^{*}\right)$ orbitals. This enables the separation in $\sigma$ and $\pi$ contributions in eqs. (1) through (3) of the total polarizability $\alpha$ and of the hyperpolarizabilities $\beta$ and $\gamma$. In Figures 2 through 4, the $\sigma$ and $\pi$ contributions to the total largest components $\alpha_{y y}$ $\beta_{y y y^{\prime}}$ and $\gamma_{y y y y}$ of the six molecules under investigation are shown.

In Figure 2, the behavior of $\alpha_{y y}^{\sigma}, \alpha_{y y}^{\pi}$, and $\alpha_{y y}^{\text {tot }}$ clearly shows that the contributions of the donor (D), spacer (II), and acceptor (A) moieties of the various molecules to the polarizability seem to be additive. For instance, by subtracting $\alpha_{y y}^{\text {tot }}$ of benzene from $\alpha_{y y}^{\text {tot }}$ of nitrobenzene or phenol, one obtains the polarizability $\alpha$ of the nitro group or of the hydroxyl group, respectively. It can also easily be seen that the polarizability of each di-substituted benzene could approximately be obtained by adding the polarizability of nitrobenzene and phenol and by subtracting the polarizability of benzene. Moreover, the di-substituted molecules possess, in magnitude, approximately the same polarizability components. This leads to the conclusion that the linear polarizability is not a function of the position of the substituents but is mainly a function of their chemical character, and that the $D, \Pi$, and A groups respond independently to the electric field. This is in agreement with the results of Velders et al., ${ }^{7}$ who showed that the linear polarizability of para-nitroaniline is only weakly dependent on the relative rotations of the substituents.

In Figure 3, the $\sigma$ and $\pi$ contributions to the total $\beta_{y y y}$ are displayed. Concerning the $\sigma$ orbitals it is observed that, with the exception of phenol, each $\sigma$ contribution is negative and that the $\beta_{y y y}^{\sigma}$ of the di-substituted compounds consists of the contribution of each of the constituting groups. While the $\sigma$ and the $\pi$ molecular orbitals were contributing almost equally to the linear polarizabiltiy, $\alpha_{y y}$, their contributions to the nonlinear polarizability $\beta_{y y y}$ differ substantially. The $\pi$ molecular orbitals mainly determine the $\beta_{y y y}^{\text {tot }}$ components. The relative position of the substituents affects, on the other hand, $\beta_{y y y}^{\pi}$ and not $\beta_{y y y}^{\sigma}$. This again is in line with the results obtained for para-nitroaniline. ${ }^{7}$ We notice that the calculated $\beta_{y y y}^{\text {tot }}$ components of the di-substituted ben-

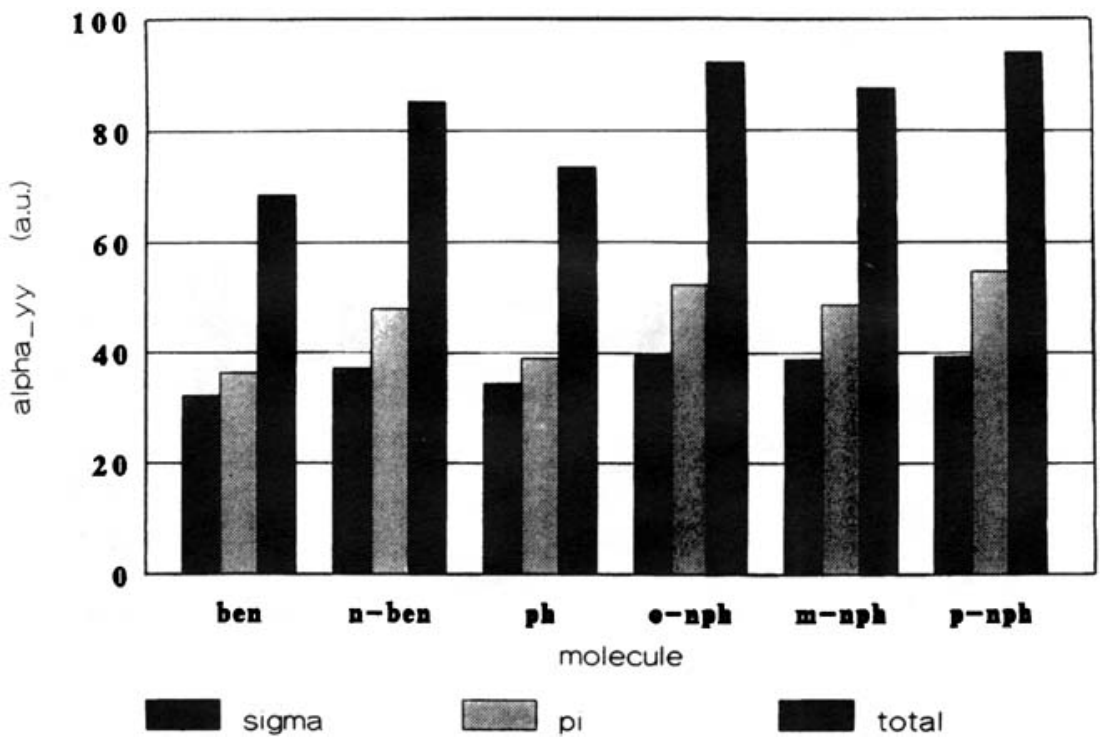

FIGURE 2. The $\sigma$ and $\pi$ molecular orbital contributions to the largest components of the linear polarizability $\alpha$ of benzene (ben), nitrobenzene (n-ben), phenol (ph), ortho-nitrophenol [o-nph(-a)], meta-nitrophenol (m-nph), and para-nitrophenol (p-nph). 


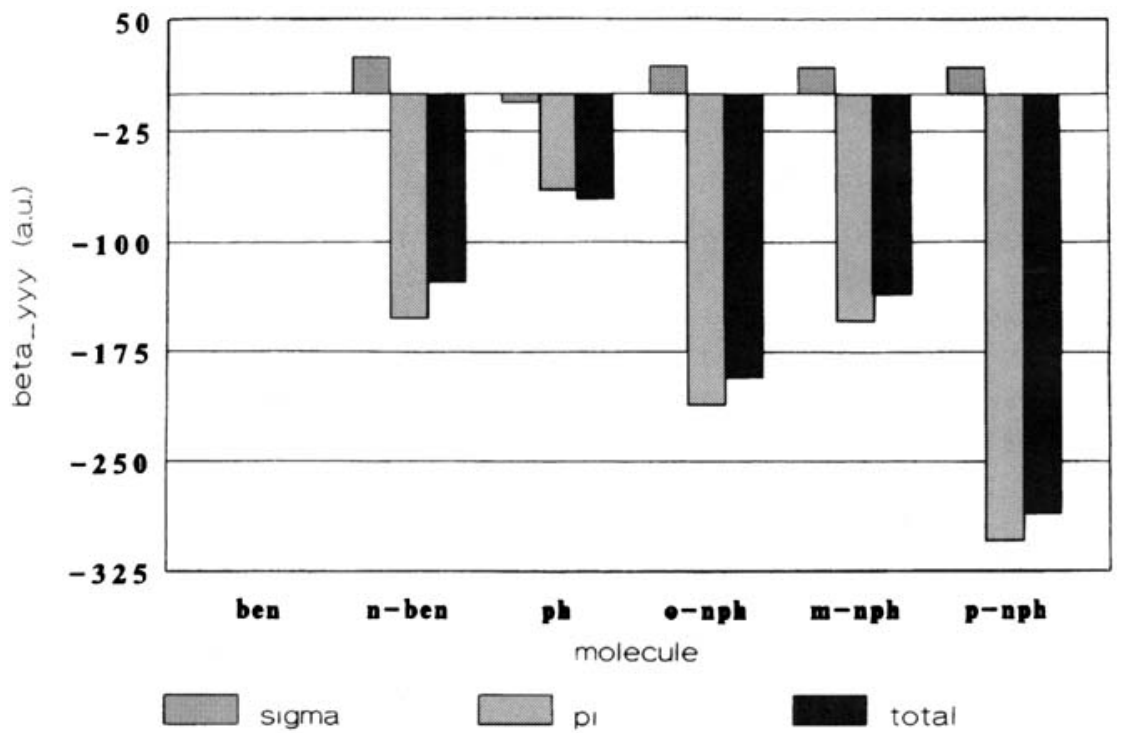

FIGURE 3. The $\sigma$ and $\pi$ molecular orbital contributions to the largest components of the nonlinear polarizability $\beta$ of benzene (ben), nitrobenzene ( $\mathrm{n}$-ben), phenol (ph), ortho-nitrophenol [o-nph(-a)], meta-nitrophenol (m-nph), and para-nitrophenol ( $\mathrm{p}$-nph). The scale has been enlarged by a factor of 2 .

zenes are in agreement with the experimental trend and that the $\beta_{y y y}^{\text {tot }}$ component of nitrobenzene is larger than $\beta_{y}^{\text {tot }}$. The histogram consequently shows that mainly charge transfer effects characterize the first hyperpolarizability $\beta$.

Finally, in Figure 4 the $\sigma$ and the $\pi$ contributions to $\gamma_{y y y y}$ are shown. Analogously to $\beta_{y y y}$, the $\pi$ molecular orbitals contribute predominantly to the $\gamma_{y y y y}^{\text {tot }}$ components, while the $\sigma$ molecular orbitals contributions are small and approximately equal in each molecule. An additive trend is observed among the $\gamma_{y y y y}^{\sigma}$ and the $\gamma_{y y y y}^{\pi}$ components of the di-substituted molecules with respect to the corresponding $\gamma_{y_{y y y}}^{\sigma}$ and $\gamma_{y y y y}^{\pi}$ components of

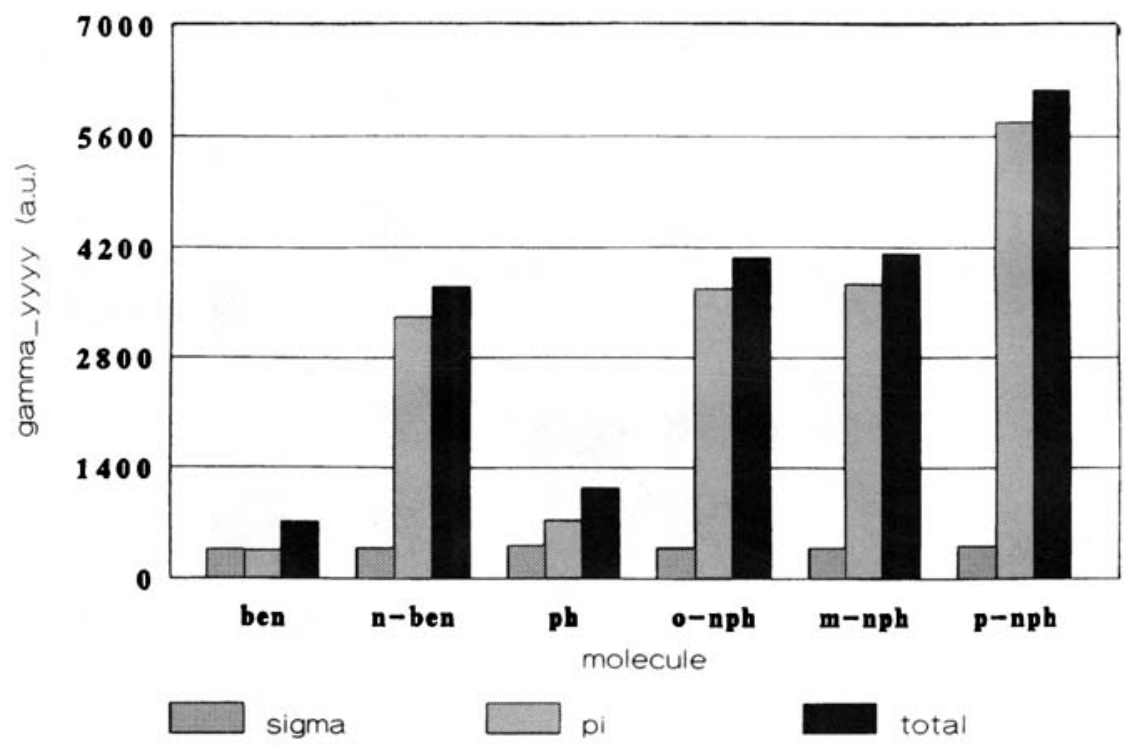

FIGURE 4. The $\sigma$ and $\pi$ molecular orbital contributions to the largest components of the nonlinear polarizability $\gamma$ of benzene (ben), nitrobenzene (n-ben), phenol (ph), ortho-nitrophenol [o-nph(-a)], meta-nitrophenol (m-nph), and para-nitrophenol (p-nph). The $\gamma$ components do not include the $K$ factor. 
benzene, phenol, and nitrobenzene. Unlike in the case of the linear polarizability, however, the relative position of the substituents determines the magnitude of the $\gamma_{y y y y}$ components. We can conclude that the second hyperpolarizability is determined by two additive factors: the size and the shape of the constituting groups and the charge transfer between the groups. Finally, it is seen that the calculated $\gamma_{y y y y}^{t o t}$ components of the disubstituted compounds follow the experimental trend.

The separation in $\sigma$ and $\pi$ contributions can further be analyzed in terms of each individual $\pi$ molecular orbital contribution. As can be observed in Tables V and VI, in benzene the two highest occupied molecular orbitals, which are degenerate, contribute equally to the component $\alpha_{y y}$ of the

\section{TABLE $V$.}

Individual $\pi$ Orbital Contributions to the Largest Components of the (Hyper)Polarizabilities.

\begin{tabular}{|c|c|c|c|}
\hline \multicolumn{4}{|c|}{ Benzene } \\
\hline$\pi$ & $\alpha_{y y}$ & $\beta_{y y y}$ & $\gamma_{y y y y}$ \\
\hline 17 & 0.12 & 0.00 & -17.64 \\
\hline 20 & 18.20 & 0.00 & 677.96 \\
\hline 21 & 18.05 & 0.00 & -307.28 \\
\hline$\pi$-tot & 36.37 & 0.00 & 353.04 \\
\hline$\sigma$-tot & 32.17 & 0.00 & 363.14 \\
\hline Total & 68.54 & 0.00 & 716.18 \\
\hline \multicolumn{4}{|c|}{ Phenol } \\
\hline$\pi$ & $\alpha_{y y}$ & $\beta_{y y y}$ & $\gamma_{y y y y}$ \\
\hline 19 & 0.44 & -1.60 & 58.68 \\
\hline 23 & 0.20 & -2.46 & -33.25 \\
\hline 24 & 17.75 & -3.78 & -283.14 \\
\hline 25 & 20.64 & -24.76 & 991.74 \\
\hline$\pi$-tot & 39.03 & -32.59 & 734.03 \\
\hline$\sigma$-tot & 34.47 & -2.96 & 414.35 \\
\hline Total & 73.50 & -35.54 & 1148.38 \\
\hline \multicolumn{4}{|c|}{ Nitrobenzene } \\
\hline$\pi$ & $\alpha_{y y}$ & $\beta_{y y y}$ & $\gamma_{y y y y}$ \\
\hline 19 & 0.95 & 4.22 & -0.04 \\
\hline 26 & 0.58 & 5.01 & -336.52 \\
\hline 30 & 0.19 & 1.54 & 36.97 \\
\hline 31 & 28.49 & -87.87 & 3854.79 \\
\hline 32 & 17.78 & 1.17 & -233.89 \\
\hline$\pi$-tot & 47.99 & -75.94 & 3321.31 \\
\hline$\sigma$-tot & 37.17 & 12.33 & 383.20 \\
\hline Total & 85.16 & -63.61 & 3704.51 \\
\hline
\end{tabular}

The total $\pi$ and $\sigma$ contributions are also shown. $\alpha_{y y}, \beta_{y y y}$, and $\gamma_{y y y y}$ are given in au. The calculation has been performed at the TZV basis set level. linear polarizability, while they contribute differently in magnitude and in sign to the $\gamma_{y y y y}$ component.

In phenol, the two highest occupied molecular orbitals, orbitals 24 and 25 , correspond to the asymmetric combination of the $p_{z}$ atomic orbitals of the ring carbon atoms and to the asymmetric combination of the $p_{z}$ atomic orbitals of the ring carbon atoms and of the hydroxyl oxygen atom, respectively. They contribute similarly to $\alpha_{y y}$, differently in magnitude to $\beta_{y y y}$, and, finally, differently in sign and magnitude to $\gamma_{y y y y}$. These differences can be related to the participation of the $p_{z}$ orbital of oxygen in the molecular orbital 25. $\alpha_{y y}^{25}, \alpha_{y y}$ of orbital 25 , is larger than $\alpha_{y y}^{24}$, since the contribution of the $p_{z}$ orbital of oxygen affects the size and the shape of molecular orbital 25 . Concerning the first hyperpolarizability, the charge transfer effects due to the $p_{z}$ atomic orbitals of the ring carbon atoms are basically negligible $\left(\beta_{y y y}^{24}\right)$, while the participation of the $p_{z}$ atomic orbital of the hydroxyl oxygen in $\beta_{y y y}^{25}$ increases the effect by one order of magnitude. A similar trend is observed for the $\gamma_{y y y y}$ component, where $\gamma_{y y y y}^{25}$ is more than three times larger in magnitude than $\gamma_{y y y y}^{24}$, but, in this case, with the opposite sign. Orbitals 19 and 23 contribute significantly only to $\gamma_{y y y y}^{\pi}$.

The analysis of nitrobenzene can be performed analogously. Molecular orbitals 31 and 32 are mainly contributing to the total linear polarizability $\alpha_{y y}^{\pi}$, while $\beta_{y y y}^{\pi}$ and $\gamma_{y y y y}^{\pi}$ have dominant contributions from orbital 31 . Orbitals 19,26 , and 30 contribute more than orbital 32 to $\beta_{y y y}^{*}$, while $\gamma_{y y y y}^{26}$ is larger than $\gamma_{y_{y y y}}^{32}$. As the molecular orbital coefficients matrix shows, orbital $31, \pi^{31}$, is an asymmetric combination of the $p_{z}$ atomic orbitals of the ring carbon atoms with the $p_{z}$ atomic orbitals of the nitro oxygen atoms, while $\pi^{32}$ is the asymmetric combination of the $p_{z}$ atomic orbitals of the ring carbon atoms. As for phenol, the effect of charge transfer among only the $p_{z}$ orbitals of the ring carbons is negligible in $\beta$ and small in $\gamma$, if the $p_{z}$ orbitals of the substituent groups do not contribute to the molecular orbitals. Orbitals 19 and 30 are the symmetric and antisymmetric molecular orbitals of the nitro group, respectively. They contribute to $\beta$, but their contributions to the $\gamma$ values are not significant.

In Tables VII and VIII, the individual $\pi$ orbital contributions to the linear and nonlinear polarizabilities of the di-substituted compounds are given. In all three molecules, the major contributions to the $\alpha_{y y}, \beta_{y y y}$, and $\gamma_{y y y y}$ components are given by 
TABLE VI.

TZV Molecular Orbitals Coefficient Matrix of the $\pi$ Occupied Orbitals.

\begin{tabular}{|c|c|c|c|c|c|c|c|c|c|c|}
\hline \multicolumn{11}{|c|}{ Benzene } \\
\hline$\pi$ & $c_{1}$ & $c_{2}$ & $c_{3}$ & $\mathrm{C}_{4}$ & $c_{5}$ & $c_{6}$ & & & & \\
\hline 17 & 0.2 & 0.2 & 0.2 & 0.2 & 0.2 & 0.2 & & & & $\pi_{\text {ring }}$ sym \\
\hline 20 & 0.1 & -0.1 & -0.3 & -0.1 & 0.1 & 0.3 & & & & $\pi_{\text {ring }}$ asym \\
\hline 21 & 0.2 & 0.2 & 0.0 & -0.2 & -0.2 & 0.0 & & & & $\pi_{\text {ring }}$ asym \\
\hline \multicolumn{11}{|c|}{ Phenol } \\
\hline$\pi$ & $\mathrm{c}_{2}$ & $c_{3}$ & $\mathrm{c}_{4}$ & $c_{5}$ & $c_{6}$ & $c_{7}$ & $o_{12}$ & & & \\
\hline 19 & 0.1 & 0.1 & 0.1 & 0.1 & 0.1 & 0.2 & 0.4 & & & $\pi_{\mathrm{OH}+\text { ring }}$ sym \\
\hline 23 & -0.2 & -0.2 & -0.2 & -0.1 & -0.1 & -0.1 & 0.3 & & & $\pi_{\mathrm{OH}+\text { ring }}$ asym \\
\hline 24 & 0.0 & -0.2 & 0.2 & -0.2 & 0.2 & 0.0 & 0.0 & & & $\pi_{\text {ring }}$ asym \\
\hline 25 & 0.3 & 0.1 & 0.1 & -0.1 & -0.2 & -0.2 & 0.2 & & & $\pi_{\mathrm{OH}+\text { ring }}$ asym \\
\hline \multicolumn{11}{|c|}{ Nitrobenzene } \\
\hline$\pi$ & $c_{2}$ & $c_{3}$ & $\mathrm{c}_{4}$ & $c_{5}$ & $c_{6}$ & $c_{7}$ & $n_{1}$ & $o_{13}$ & $o_{14}$ & \\
\hline 19 & 0.1 & 0.0 & 0.0 & 0.0 & 0.0 & 0.0 & 0.3 & 0.2 & 0.2 & $\pi_{\mathrm{NO}_{2}}$ sym \\
\hline 26 & 0.2 & 0.2 & 0.2 & 0.2 & 0.2 & 0.2 & -0.1 & -0.1 & -0.1 & $\pi_{\mathrm{NO}_{3}+\text { ring }}^{2}$ asym \\
\hline 30 & 0.0 & 0.0 & 0.0 & 0.0 & 0.0 & 0.0 & 0.0 & 0.4 & -0.4 & $\pi_{\mathrm{NO}_{2}}$ asym \\
\hline 31 & -0.3 & -0.1 & -0.1 & 0.1 & 0.1 & 0.3 & 0.0 & 0.1 & 0.1 & $\pi_{\mathrm{NO}_{3}+\text { ring }}$ asym \\
\hline 32 & 0.0 & 0.2 & -0.2 & 0.2 & -0.2 & 0.0 & 0.0 & 0.0 & 0.0 & $\pi_{\text {ring }}$ asym \\
\hline
\end{tabular}

molecular orbitals 35 and 36 . A very interesting trend can be observed: By going from the ortho to the para isomers, the magnitude of the contributions to the $\alpha_{y y}, \beta_{y y y}$, and $\gamma_{y y y y}$ components decreases in $\pi^{35}$ and increases in $\pi^{36}$. This can be distinctly observed for all components and, in particular, for $\beta_{y y y}$ and $\gamma_{y y y y}$. If we look at the molecular orbital coefficients matrix, we see that in the ortho and in the meta isomers, orbital 35 is the asymmetric combination of the $p_{z}$ orbitals of the ring carbon atom and of the nitro oxygen atoms and that orbital 36 is due to the asymmetric combination of the $p_{z}$ orbitals of the ring carbon atoms and of the $p_{z}$ orbital of the hydroxyl oxygen atom, with only a minor contribution of the $p_{z}$ orbitals of the nitro oxygen atoms. In para-nitrophenol, on the contrary, orbital 35 is only built up from the $p_{z}$ atomic orbitals of the ring carbon atoms, while orbital 36 is an asymmetric combination of all $p_{z}$ atomic orbitals except the $p_{z}$ orbital of the nitrogen atom. The para position is, therefore, geometrically favorable to the contribution of almost all $p_{z}$ atomic orbitals. This can be observed starting from the linear polarizability components, which redistribute between orbitals 35 and 36 . The largest effects are observed in $\beta$ and $\gamma$, where the maximum orbital spatial distribution enhances considerably the charge transfer phenomenon. Orbitals
27 and 31 also contribute significantly to the total $\pi$ components. They correspond to the antisymmetric combinations of the $p_{z}$ ring carbon atom orbitals with the $p_{z}$ orbitals of the nitro group and of the hydroxyl oxygen atom. It is noteworthy that the $p_{z}$ orbitals of the nitro group mix into orbital 27 of the ortho isomer, while in the meta and para isomers it is orbital 31 which shows such a property, indicating that in the ortho isomer the mixture of the $p_{z}$ atomic orbitals of the nitro group into the $\pi_{\mathrm{OH}+\text { ring }}$ orbital makes orbital 31 more stable than the orbital $\pi_{\mathrm{OH}+\text { ring }}$ only.

We now turn our discussion to a specific type of approximation which can be performed within the SOO approach. Previously, it has been attempted to describe the nonlinear hyperpolarizabilities in terms of the empirical TLM. ${ }^{27}$ The idea of this model is based on the assumption that only two states determine the magnitude and the sign of the nonlinear mechanisms: the electronic ground state and the lowest excited electronic state, or charge transfer state, with the neglect of all other possible interactions between the ground state and the remaining electronic excited states and the interactions among the electronic excited states themselves. This empirical approach is generally considered satisfactory within the limits of the approximations involved. Within the SOO formal- 
TABLE VII.

Individual $\pi$ Occupied Orbital Contributions to the

Largest Components of the (Hyper)Polarizabilities.

\begin{tabular}{|c|c|c|c|}
\hline \multirow[b]{2}{*}{$\pi$-orb } & \multicolumn{2}{|c|}{ o-Nitrophenol } & \multirow[b]{2}{*}{$\gamma_{y y y y}$} \\
\hline & $\alpha_{y y}$ & $\beta_{y y y}$ & \\
\hline 22 & 0.72 & 4.11 & -3.33 \\
\hline 27 & 0.87 & 6.58 & -122.27 \\
\hline 31 & 1.16 & -5.79 & -275.90 \\
\hline 34 & 0.52 & 0.95 & 78.13 \\
\hline 35 & 28.14 & -90.35 & 3545.98 \\
\hline 36 & 21.03 & -21.16 & 455.59 \\
\hline$\pi$-tot & 52.44 & -105.67 & 3678.20 \\
\hline$\sigma$-tot & 39.72 & 9.29 & 389.86 \\
\hline Total & 92.16 & -96.38 & 4068.06 \\
\hline \multicolumn{4}{|c|}{ m-Nitrophenol } \\
\hline $\begin{array}{l}\pi \text {-orb } \\
22\end{array}$ & $\begin{array}{l}\alpha_{y y} \\
0.98\end{array}$ & $\begin{array}{r}\beta_{y y y} \\
4.23\end{array}$ & $\gamma_{y y y y}$ \\
\hline 27 & 0.55 & -2.61 & -65.02 \\
\hline 31 & 1.50 & 14.38 & -390.41 \\
\hline 34 & 0.22 & 1.62 & 37.79 \\
\hline 35 & 24.42 & -68.23 & 3038.74 \\
\hline 36 & 21.06 & -26.27 & 1112.92 \\
\hline$\pi$-tot & 48.73 & -76.87 & 3736.11 \\
\hline$\sigma$-tot & 38.86 & 9.01 & 386.65 \\
\hline Total & 87.59 & -67.87 & 4122.76 \\
\hline \multicolumn{4}{|c|}{ p-Nitrophenol } \\
\hline$\pi$-orb & $\alpha_{y y}$ & $\beta_{y y y}$ & $\gamma_{y y y y}$ \\
\hline 23 & 0.85 & 4.31 & -13.68 \\
\hline 27 & 0.57 & -2.24 & 142.49 \\
\hline 31 & 0.80 & 4.27 & -628.01 \\
\hline 34 & 0.18 & 1.38 & 31.18 \\
\hline 35 & 17.53 & -3.93 & -127.17 \\
\hline 36 & 34.80 & -155.80 & 6370.99 \\
\hline$\pi$-tot & 54.73 & -152.01 & 5775.80 \\
\hline$\sigma$-tot & 39.32 & 9.15 & 410.78 \\
\hline Total & 94.05 & -142.86 & 6186.58 \\
\hline
\end{tabular}

The total $\pi$ and $\sigma$ contributions are also shown. $\alpha_{y y}, \beta_{y y y}$, and $\gamma_{y y y y}$ are given in au. The calculations have been performed at the TZV basis set level.

ism, the TLM reduces to two molecular orbitals: the highest occupied molecular orbital (HOMO) and the lowest unoccupied molecular orbital (LUMO).

Retaining only two molecular orbitals, the occupied $a$ and the virtual $r$, the TLM expressions for $\beta$ and $\gamma$ can easily be derived from eqs. (2) and (3) for the static case. The expression of $\beta$ reduces to

$$
\beta_{y y y}=-2 \frac{\langle y\rangle_{a r}^{2}}{\left(\epsilon_{r}-\epsilon_{a}\right)^{2}}\left(\langle y\rangle_{r r}-\langle y\rangle_{a a}\right)
$$

Analogously, the expression of $\gamma$ reduces to

$$
\gamma_{y y y y}=2 \frac{\langle y\rangle_{a r}^{2}}{\left(\epsilon_{r}-\epsilon_{a}\right)^{3}}\left(\left[\langle y\rangle_{r r}-\langle y\rangle_{a a}\right]^{2}-\langle y\rangle_{a r}^{2}\right)
$$

In the present work, we wish to investigate the effects that such a drastic approximation as the TLM may have on the SOO approach. In Table IX, we show the 6-31G $\beta_{y y y}$ and $\gamma_{y y y y}$ components of the nitrophenols and nitrobenzene, calculated at three different levels of approximation: $\beta_{y y y}^{5 O O}$ and $\gamma_{y_{y y y}}^{\mathrm{SOO}}$ are the calculated SOO $\beta_{y y y}$ and $\gamma_{y y y y} \mathrm{com}-$ ponents; $\beta_{y y y}^{a}$ and $\gamma_{y y y y}^{a}$ have been obtained by truncating the Sum Over Orbitals expressions and retaining only terms which involve transitions between the $\pi^{a}$ occupied orbital and the $\pi^{r}$ virtual orbitals and the transitions between the $\pi^{r}$ and the $\pi^{s}$ virtual orbitals; and, finally, $\beta_{y y y}^{c t}$ and $\gamma_{y y y y}^{c t}$ have been obtained via the TLM $(c t=$ charge transfer) (i.e., they involve only the transitions between the two orbitals $a$ and $r$ and the transitions between themselves).

As can be observed in Table IX, the neglect of the transitions involving different occupied orbitals and the transitions involving different occupied orbitals and the same virtual orbital, as in $\beta_{y y y}^{a}$, affects the $\beta_{y y y}^{S O O}$ values by approximately $4 \%$ on the average for the nitrophenols and by approximately $37 \%$ for nitrobenzene. The neglect of all terms involving transitions among different virtual orbitals and the transitions between different virtual orbitals and the same occupied orbital, as in $\beta_{y y y}^{c t}$, results in a value which is $90 \%$ larger than the $\beta_{y y y}^{500}$ value. In the case of the second hyperpolarizability, the $\gamma_{y y y y}^{a}$ overestimates or underestimates the corresponding $\gamma_{y y y y}^{S O O}$ by about $10 \%$, while the charge transfer $\gamma_{y y y y}^{c t}$ values underestimate by about $22 \%$ on the average the $\gamma_{y y y y}^{500}$ values.

If we analyze the trends, we notice that the $\beta_{y y y}^{c t}$ values reproduce the experimental trend. $\beta_{y y y}^{c t}$ of nitrobenzene is very large, and only paranitrophenol possesses a larger $\beta_{y y y}^{c t}$ value. The inclusion of the other transitions between virtual orbitals decreases all values, particularly the nitrobenzene value. The inclusion of all transitions has a negligible effect only on the ortho- and meta-nitrophenols, while the value for nitrobenzene is decreased to the largest extent.

A completely different trend characterizes $\gamma_{y y y y}$. The $\gamma_{y y y y}^{c t}$ values poorly reproduce the experimental values, while the inclusion of transitions among 
TABLE VIII.

TZV Molecular Orbital Coefficient Matrix of the $\pi$ Occupied Orbitals.

\begin{tabular}{|c|c|c|c|c|c|c|c|c|c|c|c|}
\hline \multirow[b]{2}{*}{$\pi$} & \multirow[b]{2}{*}{$\mathrm{c}_{2}$} & \multirow[b]{2}{*}{$\mathrm{c}_{3}$} & \multirow[b]{2}{*}{$\mathrm{c}_{4}$} & \multirow[b]{2}{*}{$c_{5}$} & \multirow[b]{2}{*}{$c_{6}$} & \multicolumn{2}{|c|}{ o-Nitrophenol } & \multirow[b]{2}{*}{$o_{12}$} & \multirow[b]{2}{*}{$o_{13}$} & \multirow[b]{2}{*}{$O_{14}$} & \multirow[b]{3}{*}{$\pi_{\mathrm{NO}_{2}+\text { ring }}$ sym } \\
\hline & & & & & & $c_{7}$ & $n_{1}$ & & & & \\
\hline 22 & -0.1 & -0.1 & 0.0 & 0.0 & 0.0 & 0.0 & -0.3 & -0.2 & -0.2 & 0.0 & \\
\hline 27 & 0.1 & 0.2 & 0.1 & 0.1 & 0.1 & 0.1 & -0.1 & -0.1 & -0.1 & 0.4 & $\pi_{\mathrm{NO}_{2}+\mathrm{OH}+\text { ring asym }}$ \\
\hline 31 & 0.2 & 0.0 & 0.2 & 0.1 & 0.2 & 0.2 & 0.0 & 0.0 & -0.1 & -0.3 & $\pi_{\mathrm{OH}+\text { ring }}$ asym \\
\hline 34 & 0.0 & 0.0 & 0.0 & 0.0 & 0.0 & 0.0 & 0.0 & -0.3 & 0.4 & -0.1 & $\pi_{\mathrm{OH}+\mathrm{NO}_{2}}$ asym \\
\hline 35 & -0.3 & 0.0 & -0.2 & 0.2 & 0.1 & 0.2 & 0.0 & 0.1 & 0.1 & 0.0 & $\pi_{\mathrm{NO}_{2}+\text { ring }}$ asym \\
\hline \multirow[t]{2}{*}{36} & -0.1 & -0.2 & 0.2 & -0.2 & 0.3 & 0.0 & 0.0 & 0.1 & 0.0 & 0.2 & $\pi_{\mathrm{OH}+\mathrm{NO}_{2}+\text { ring asym }}$ \\
\hline & \multicolumn{10}{|c|}{ m-Nitrophenol } & \\
\hline$\pi$ & $c_{2}$ & $\mathrm{c}_{3}$ & $\mathrm{c}_{4}$ & $\mathrm{c}_{5}$ & $c_{6}$ & $c_{7}$ & $n_{1}$ & $o_{12}$ & $o_{13}$ & $o_{14}$ & \\
\hline 22 & -0.1 & 0.0 & 0.0 & 0.0 & 0.0 & 0.0 & -0.3 & -0.2 & -0.2 & 0.0 & $\pi_{\mathrm{NO}_{2}}$ sym \\
\hline 27 & 0.1 & 0.1 & 0.1 & 0.2 & 0.1 & 0.1 & 0.0 & 0.0 & 0.0 & 0.4 & $\pi_{\mathrm{OH}+\text { ring }}$ sym \\
\hline 31 & 0.2 & 0.1 & 0.2 & 0.0 & 0.2 & 0.1 & -0.1 & -0.1 & -0.1 & -0.3 & $\pi_{\mathrm{OH}+\mathrm{NO}_{2}}+{ }_{\text {ring }}$ asym \\
\hline 34 & 0.0 & 0.0 & 0.0 & 0.0 & 0.0 & 0.0 & 0.0 & -0.4 & 0.4 & 0.0 & $\pi_{\mathrm{NO}_{2}}$ asym \\
\hline 35 & -0.2 & -0.2 & 0.0 & 0.0 & 0.2 & 0.2 & 0.0 & 0.1 & 0.1 & 0.0 & $\pi_{\mathrm{NO}_{2}+\text { ring }}$ asym \\
\hline \multirow[t]{2}{*}{36} & 0.1 & -0.2 & 0.2 & -0.2 & 0.1 & -0.1 & 0.0 & -0.1 & 0.0 & 0.2 & $\pi_{\mathrm{OH}+\mathrm{NO}_{2}+\text { ring }}$ asym \\
\hline & \multicolumn{10}{|c|}{ p-Nitrophenol } & \\
\hline$\pi$ & $\mathrm{c}_{2}$ & $\mathrm{c}_{3}$ & $\mathrm{c}_{4}$ & $c_{5}$ & $\mathrm{c}_{6}$ & $c_{7}$ & $n_{1}$ & $o_{12}$ & $\mathrm{O}_{13}$ & $o_{14}$ & \\
\hline 23 & -0.1 & 0.0 & 0.0 & 0.0 & 0.0 & 0.0 & -0.3 & -0.2 & -0.2 & 0.0 & $\pi_{\mathrm{NO}_{2}}$ sym \\
\hline 27 & 0.0 & 0.1 & 0.1 & 0.1 & 0.1 & 0.2 & 0.0 & 0.0 & 0.0 & 0.4 & $\pi_{\mathrm{OH}+\text { ring }}$ sym \\
\hline 31 & -0.2 & -0.2 & -0.2 & -0.1 & -0.1 & -0.1 & 0.1 & 0.1 & 0.1 & 0.3 & $\pi_{\mathrm{OH}+\mathrm{NO}_{2}+\text { ring }}$ asym \\
\hline 34 & 0.0 & 0.0 & 0.0 & 0.0 & 0.0 & 0.0 & 0.0 & -0.4 & 0.4 & 0.0 & $\pi_{\mathrm{NO}_{2}}$ asym \\
\hline 35 & 0.0 & -0.2 & 0.2 & -0.2 & 0.3 & 0.0 & 0.0 & 0.0 & 0.0 & 0.0 & $\pi_{\text {ring }}$ asym \\
\hline 36 & -0.3 & -0.1 & -0.1 & 0.2 & 0.1 & 0.2 & 0.0 & 0.1 & 0.1 & -0.2 & $\pi_{\mathrm{OH}+\mathrm{NO}_{2}+\text { ring }}$ asym \\
\hline
\end{tabular}

virtual orbitals improves the agreement between theory and experiment. The $\gamma_{y y y y}^{S O O}$ values produce indeed the best trend, with nitrobenzene, which is largely underestimated, being the only exception.

We will continue our discussion with the analysis based on the molecular orbitals which mostly contribute to the total components of the (hyper)polarizabilities. For this purpose, we have mapped out the occupied molecular orbitals 35, 35, and 36 or the ortho-, meta-, and para-nitrophenols, respectively, and the lowest virtual molecular orbital, orbital 37, which possesses the largest oscillator strengths with the aforementioned occupied orbitals. The molecular orbitals are shown in Figures $5 a$ and $5 b$.

We first discuss the closely related ortho and meta isomers. In the occupied orbital of both conformations, the bond that connects the hydroxyl group with the phenyl ring coincides with a nodal plane. Consequently, in neither case the $p_{z}$ orbital

TABLE IX.

Comparison between the Sum Over Orbitals (SOO) and the Two-Level Model (ct) Methods.

\begin{tabular}{|c|c|c|c|c|c|c|}
\hline & $\beta_{y y y}^{\text {SOO }}$ & $\beta_{y y y}^{a}$ & $\beta_{y y y}^{c t}$ & $\gamma_{\text {yyyy }}^{\text {SOO }}$ & $\gamma_{y y y y}^{a}$ & $\gamma_{y y y y}^{c t}$ \\
\hline nben & -61.3 & -83.7 & -155.1 & 3116.0 & 3346.6 & 2576.8 \\
\hline o-nph & -89.1 & -85.1 & -138.4 & 3429.4 & 3193.8 & 2404.2 \\
\hline m-nph & -66.1 & -66.3 & -117.3 & 3492.9 & 2648.0 & 2378.5 \\
\hline p-nph & -131.1 & -141.6 & -230.7 & 5297.0 & 5583.3 & 4921.7 \\
\hline
\end{tabular}

The calculations have been performed at the 6-31G basis set level. 
(a)

(1)

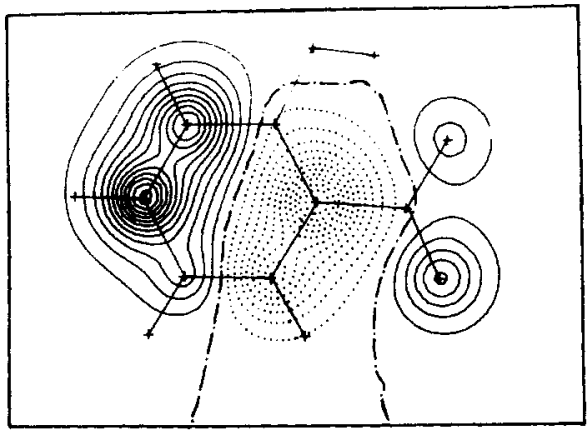

(2)

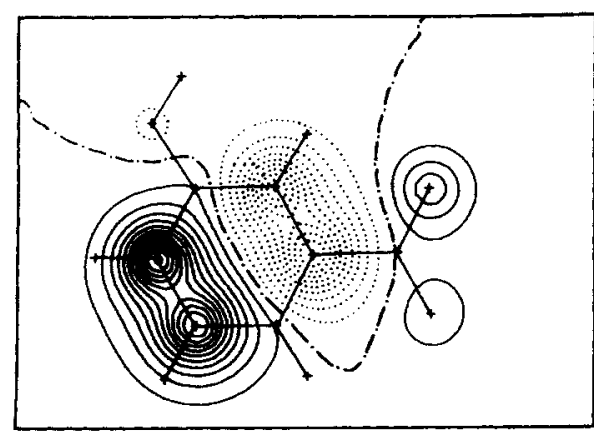

(3)

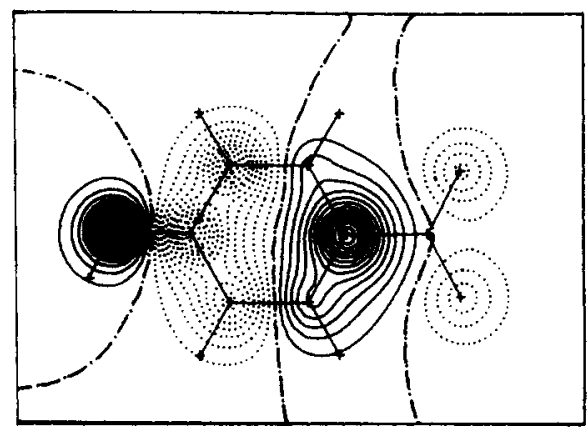

(b)

(1)

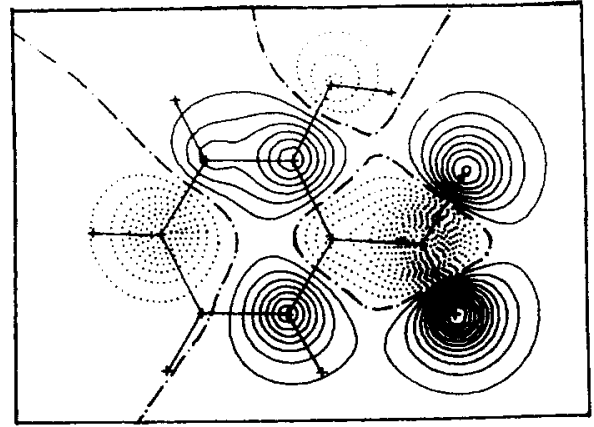

(2)

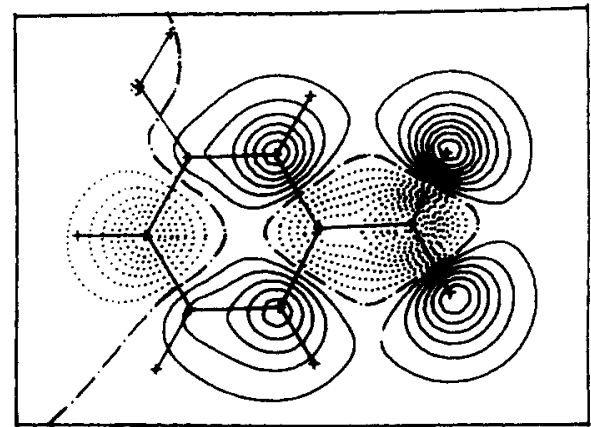

(3)

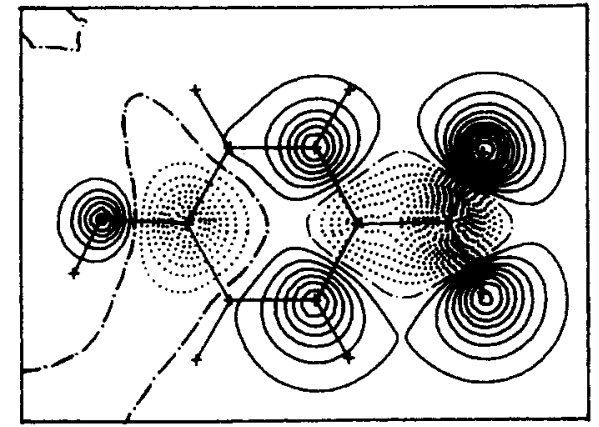

FIGURE 5. (a) Occupied $\pi$ molecular orbitals (1) 35 , (2) 35 , and (3) 36 of ortho-nitrophenol, meta-nitrophenol, and para-nitrophenol, respectively. (b) Unoccupied $\pi^{*}$ molecular orbitals (1) 37, (2) 37 , and (3) 37 of ortho-nitrophenol, meta-nitrophenol, and para-nitrophenol, respectively. The maps have been obtained by cutting the $p_{z}$ atomic orbitals with an $x y$-plane 0.05 au above the molecular plane. The contour interval amounts to $0.0148 \mathrm{e} / \AA^{3}$. Positive, zero, and negative contours are drawn as solid, dash-dotted, and dotted lines, respectively.

of the hydroxyl oxygen atom contributes to the occupied $\pi$ orbital. In fact, the $\pi$ orbitals are nearly mirror images, with a slightly larger participation of the nitro group in the ortho isomer. The lowest unoccupied $\pi$ orbital of the ortho isomer closely resembles the unoccupied $\pi$ orbital of the meta isomer, with a stronger participation of the hydroxyl and nitro oxygen atoms in the former one. The product of the orbitals of the highest occupied and lowest unoccupied orbitals constitutes the transition density in the two-orbital model. Clearly in neither case the hydroxyl oxygen atom contributes to this density and thus to the transition moment. According to Oudar et al., ${ }^{27}$ the first hyperpolarizability, $\beta$, depends on the product of the transition moment with the difference of the dipole moment in the excited state and in the ground state. The peripheral position of the nitro oxygen atoms multiplies the moderate differences in charge density of the nitro oxygen atoms between the ortho and the meta isomers into substantial differences in dipole moment, explaining 
the trend in the $\beta$. The situation of the para isomer is different. The hydroxyl oxygen atom participates considerably in both the occupied and the virtual orbitals. With all other atoms contributing as well, the highly polar transition density leads to a large transition moment. The transition is seen to result in a lower density on the hydroxyl oxygen atom and a strongly increased density on the nitro group, yielding a large shift in dipole moment. We can conclude from this discussion that the twoorbital model may provide insight into the trend of the first hyperpolarizability, though considerable caution is required in drawing definite conclusions. Unfortunately, the expression for $\gamma$ contains too many terms to allow a similar qualitative discussion, although the large transition moment of the para isomer suggests a large value for $\gamma_{y y y y}$.

It is experimentally and theoretically well established that the most stable conformation of ortho nitrophenol is intramolecularly hydrogen bonded. $^{40}$ Our 3-21G optimized geometry does confirm the experimental data and shows that at the equilibrium geometry both the nitro and the hydroxyl groups are in the molecular plane. Since in all three 3-21G optimized geometries (ortho, meta, and para) the nitro group is in the plane and since only the ortho conformation displays hydrogen bonding, we have investigated the effect of this intramolecular interaction on the linear and nonlinear polarizabilities. We have consequently looked for a local minimum corresponding to the free $\mathrm{OH}$ structure on the potential energy surface of ortho-nitrophenol. This minimum lies at about $15 \mathrm{kcal}$ (3-21G basis set) above the most stable intramolecularly hydrogen bonded conformation. The free $\mathrm{OH}$ optimized conformation is characterized by the $\mathrm{OH}$ group, which is flipped by about $180^{\circ}$ with respect to the bonded conformation, and by the nitro group, which is rotated off plane by about $17^{\circ}$, since, in this case the oxygen atom of the nitro group, which is close to the hydroxyl group, is repelled by the hydroxyl oxygen. The free $\mathrm{OH}$ optimized structure is shown in Figure 1. We name the $\mathrm{H}$-bonded and $\mathrm{H}$-unbonded conformations o-nph-a and o-nph-b, respectively. To eliminate the possible effect of the rotation of the nitro group, we have also considered another conformation, o-nph-c, which has been obtained from $o-n p h-b$, by constraining the nitro group into the molecular plane. In Table $X$ the largest components of the linear and nonlinear polarizabilities for the three different ortho conformations are shown. The first and the second hyperpolarizabilities have been calculated within the SOO and the
TABLE $X$ Effects of the Intramolecular Hydrogen Bonding and of the Rotation of the Nitro Group on the (Hyper)Polarizabilities.

\begin{tabular}{lccrcc}
\hline & $\alpha_{y y}$ & $\beta_{y y y}^{\text {soo }}$ & \multicolumn{1}{c}{$\beta_{y y y}^{c t}$} & $\gamma_{y y y y}^{\text {soo }}$ & $\gamma_{y y y y}^{c t}$ \\
\hline o-nph-a $^{\mathrm{a}}$ & 87.8 & -89.1 & -138.4 & 3429.4 & 2404.2 \\
o-nph-b $^{\mathrm{b}}$ & 82.9 & -48.0 & -97.5 & 2588.4 & 1340.0 \\
o-nph-c $^{\mathrm{c}}$ & 83.6 & -51.5 & -99.5 & 2700.5 & 1307.5 \\
\hline
\end{tabular}

The predicted Sum Over Orbitals (SOO) and the Two-Level Model (ct) hyperpolarizabilities values are compared. The calculations have been performed at the 6-31G basis set level.

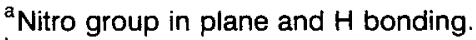

${ }^{b}$ Nitro group off plane and no $\mathrm{H}$ bonding.

${ }^{\mathrm{C}}$ Nitro group in plane and no $\mathrm{H}$ bonding.

TLM approaches. We focus first on the o-nph-b and o-nph-c conformations. It is seen that the rotation of the nitro group by $17^{\circ}$ does not affect significantly $\alpha_{y y^{\prime}}, \beta_{y y_{y}}$, and $\gamma_{y y y y} . \beta_{y y y}^{S O O}$ and $\gamma_{y y y y}^{5 O O}$ can be concluded, however, to be more sensitive to the structural changes. The differences between the o-nph-a and the o-nph-c conformations are more remarkable. $\beta_{y y y}^{S O O}$ and $\gamma_{y y y y}^{S O O}$ decrease by more than $40 \%$ and $20 \%$, respectively, while the trend is reversed in $\beta_{y y y}^{c t}$ and $\gamma_{y y y y}^{c t}$. Since the SOO values are the most accurate ones, one can conclude that the first hyperpolarizability is affected by the presence or the absence of the hydrogen bond more than the second hyperpolarizability is. When the hydrogen bond is present, the $\beta$ and $\gamma$ values are the largest ones. This can be explained by observing that the hydrogen bond has the effect of lowering the energy of the lowest unoccupied molecular orbital. From the TLM expressions of $\beta$ and $\gamma$, we can easily see that if $\Delta \epsilon_{a r}$ decreases, $\beta$ becomes larger. When the HOMO-LUMO energy difference decreases, the oscillator strength of the transition increases and $\beta$ is enhanced. This analysis, however, becomes more difficult in the case of $\gamma$, since $\gamma$ is also proportional to the difference between the change of the dipoles of the HOMO-LUMO and their oscillator strength. This difference seems to counterbalance the enhancement due to the energy differences with a resulting reduced net effect on the second hyperpolarizability.

\section{Conclusions}

In the present investigation, we have analyzed the performance of the Sum Over Orbitals (SOO) 
method by means of calculations of the linear and nonlinear polarizabilities of nitrophenols. The SOO method invokes many approximations, among which are the neglect of the coupling of the perturbation and the replacement of states energies by orbital energies. The comparison of the SOO results with the outcome of the finite field calculations shows this approximation to be satisfactory. The use of other approximations, such as the neglect of the electron correlation, of the environmental effects, and of the vibrational contributions, does not seem to destroy the validity for predicting the experimental trends. At the same time, the transparent structure of the method allows the interpretation of the linear and nonlinear molecular properties in terms of the contributions of the individual orbitals. Finally, the important advantage of the SOO method is that it can easily be applied to medium- to large-sized molecules, which are hard or impossible to handle with Coupled Hartree-Fock techniques. Molecular systems larger than nitrophenols are presently under investigation.

\section{Acknowledgments}

This work was sponsored by the Stichting Nationale Computerfaciliteiten (National Computing Facilities Foundation, NCF) for the use of supercomputer facilities, with financial support from the Nederlandse Organisatie voor Wetenschappelijk Onderzoek (Netherlands Organization for Scientific Research, NWO). The authors wish to thank the referees for their indispensable comments. One of the authors, R. B., wishes to thank Dr. L. C. H. van Corler of the Stichting Academisch Rekencentrum Amsterdam (SARA) for his assistance and Dr. J. H. van Lenthe for adjusting repeatedly the CRAY version of GAMESS to the required computational size of the present project.

\section{References}

1. J. Zyss and D. S. Chemla, Nonlinear Optical Properties of Organic Molecules and Crystals, Vols. 1 and 2, Academic Press, New York, 1987.

2. P. N. Butcher and D. Cotter, The Elements of NonLinear Optics, Cambridge University Press, Cambridge, UK, 1990

3. See Chemical Review, 94 (1994).

4. H. A. Kurtz, J. J. P. Stewart, and K. M. Dieter, J. Comp. Chem., 11, 82 (1990)
5. S. P. Karna, Z. Laskowski, G. B. Talapatra, and P. N. Prasad, J. Phys. Chem., 95, 6508 (1991).

6. G. Maroulis, J. Chem. Phys., 97, 4188 (1992).

7. G. J. M. Velders, J.-M. Gillet, P. J. Becker, and D. Feil, I. Phys. Chem., 95, 8601 (1991).

8. D. Beljonne, Z. Shuai, and J. L. Brédas, J. Chem. Phys., 98, 8819 (1993).

9. W. A. Parkinson and W. A. Zerner, J. Chem. Phys., 94, 478 (1991).

10. S. D. Di Bella, M. A. Ratner, and T. J. Marks, J. Am. Chem. Soc., 114, 5842 (1992).

11. B. M. Pierce, J. Chem. Phys., 91, 791 (1989).

12. J. E. Rice, R. D. Amos, S. M. Colwell, N. C. Handy, and J. Sanz, J. Chem. Phys., 93, 8828 (1990).

13. H. Hettema, P. E. S. Wormer, P. Jørgensen, H. J. Aa. Jensen, and T. Helgaker, J. Chem. Phys., 100, 1297 (1994).

14. S. P. Karna, P. N. Prasad, and M. Dupuis, J. Chem. Phys., 94, 1171 (1991).

15. F. Sim, S. Chin, M. Dupuis, and J. E. Rice, J. Phys. Chem., 97, 1158 (1993).

16. Y. Luo, H. Ågren, O. Vahtras, and P. Jørgensen, Chem. Phys. Lett., 207, 190 (1993)

17. H. Sekino and R. J. Bartlett, J. Chem. Phys., 98, 3022 (1993).

18. J. Waite and M. G. Papadopoulos, J. Phys. Chem., 93, 43 (1989).

19. J. Guan, P. Duffy, J. T. Carter, D. P. Chong, K. C. Casida, M. E. Casida, and M. Wrinn, J. Chem. Phys., 98, 4753 (1993).

20. D. Bishop, Rev. Mod. Phys., 62, 343 (1990).

21. B. J. Orr and J. F. Ward, Mol. Phys., 20, 513 (1971).

22. P. W. Langhoff, M. Karplus, and R. P. Hurst, J. Chem. Phys., 44, 505 (1966).

23. M. Tomonari, N. Ookubo, T. Takada, M. W. Feyereisen, and J. Almlöf, Chem. Phys. Lett., 203, 603 (1993).

24. M. Tomonari, N. Ookubo, and T. Takada, Chem. Phys. Lett., 215, 45 (1993).

25. J. Zyss, J. Chem. Phys., 70, 3341 (1979).

26. J. Zyss, J. Chem. Phys., 71, 909 (1979).

27. J. L. Oudar and D. S. Chemla, J. Chem. Phys., 66, 2664 (1977).

28. A. Szabo and N. S. Ostlund, Modern Quantum Chemistry, Macmillan, New York, 1982.

29. C. Murray and E. R. Davidson, Int. J. Quantum Chem., 43, 755 (1992).

30. Unpublished results.

31. E. R. Davidson and D. Feller, Chem. Rev., 86, 681 (1986).

32. M. Dory, L. Beudels, J. G. Fripiat, J. Delhalle, J. M. André, and M. Dupuis, Int. J. Quantum Chem., 42, 1577 (1992).

33. B. L. Hammond and J. E. Rice, J. Chem. Phys., 97, 1138 (1992).

34. T. H. Dunning, J. Chem. Phys., 53, 2823 (1970).

35. T. H. Dunning, J. Chem. Phys., 55, 716 (1971).

36. A. D. McLean and G. S. Chandler, J. Chem. Phys., 72, 5639 (1980).

37. J. S. Binkley, J. A. Pople, and W. J. Hehre, J. Am. Chem. Soc., 102, 939 (1980). 
38. W. J. Hehre, L. Radom, P. v. R. Schleyer, and J. A. Pople, Ab Initio Molecular Orbital Theory, John Wiley \& Sons, New York, 1986.

39. P. Politzer, J. M. Seminario, and P. R. Bolduc, Chem. Phys. Lett., 158, 463 (1989).

40. K. B. Borisenko, C. W. Bock, and I. Hargittai, J. Phys. Chem., 98, 1442 (1994).

41. C. Møller and M. S. Plesset, Phys. Rev., 46, 618 (1934).

42. (a) M. Dupuis, D. Spangler, and J. J. Wendoloski, NRCC Software Catalog, Vol. 1, Program N., QG01 (GAMESS), 1980; (b) M. F. Guest and J. Kendrick, GAMESS Users Manual, Daresbury Laboratory, Daresbury, UK, 1985.
43. M. W. Schmidt, K. K. Baldridge, J. A. Boatz, S. T. Elbert, M. S. Gordon, J. H. Jensen, S. Koseki, N. Matsunaga, K. A. Nguyen, S. J. Su, T. L. Windus, M. Dupuis, and J. A. Montgomery. GAMESS (Dakota), Iowa State University, 1994.

44. C. Daniel and M. Dupuis, Chem. Phys. Lett., 171, 209 (1990).

45. H. D. Cohen and C. C. Roothaan, J. Chem. Phys., 43, 534 (1965).

46. L.-T. Cheng, W. Tam, S. H. Stevenson, G. R. Meredith, G. Rikken, and S. R. Marder, J. Phys. Chem., 95, 10631 (1991). 